\title{
Human umbilical cord mesenchymal stem cells inhibit proliferation of hepatic stellate cells in vitro
}

\author{
LI-TING ZHANG $^{1 *}$, XUE-BIN PENG ${ }^{*}$, XUE-QIN FANG ${ }^{2}$, JUN-FENG LI $^{1}$, \\ $\mathrm{HONG} \mathrm{CHEN}^{1}$ and XIAO-RONG MAO ${ }^{1}$ \\ ${ }^{1}$ Department of Infectious Diseases, The First Hospital of Lanzhou University, Lanzhou, Gansu 730000; \\ ${ }^{2}$ Department of Infectious Diseases, The Central Hospital of Baoji, Baoji, Shannxi 721008, P.R. China
}

Received July 1, 2016; Accepted January 22, 2018

DOI: $10.3892 /$ ijmm.2018.3500

\begin{abstract}
The effect of human umbilical cord mesenchymal stem cells (hUC-MSCs) on the proliferation of hepatic stellate cells (HSCs) is largely unknown. The purpose of this study was to explore the mechanism of action of hUC-MSCs on the proliferation of HSCs in vitro. The upper and lower double-cell co-culture system was established between hUC-MSCs and HSCs in the experimental group. HSCs were cultured alone as a negative control group. Cell proliferation and apoptosis were determined by 3-(4,5-dimethylthiazol-2-yl)-2,5-diphenyltetrazolium bromide (MTT) assay and flow cytometry, respectively. Cell supernatants were harvested to determine the concentration of transforming growth factor- $\beta 1$ (TGF- $\beta 1)$ by ELISA. mRNA and protein of TGF- $\beta 1$, Smad 3 and Smad 7 in HSCs were determined by reverse transcription-polymerase chain reaction and western blotting, respectively. In the co-culture group, the proliferation of HSCs was significantly inhibited
\end{abstract}

Correspondence to: Dr Li-Ting Zhang or Dr Xue-Bin Peng, Department of Infectious Diseases, The First Hospital of Lanzhou University, 1 West Donggang Road, Lanzhou, Gansu 730000, P.R. China

E-mail: 1cheneye@163.com

E-mail: baomihua119@163.com

\section{"Co-first authors}

Abbreviations: MSC, mesenchymal stem cells; hUC-MSCs, human umbilical cord mesenchymal stem cells; BMSCs, bone marrow mesenchymal stem cells; ECM, extracellular matrix; TGF- $\beta$, transforming growth factor- $\beta$; MTT, 3 -(4,5-dimethylthiazol2-2yl)-2,5-diphenyltetrazolium bromide; ELISA, enzyme linked immunosorbent assay; PBS, phosphate-buffered saline; FBS, fetal bovine serum; DMEM-LG, Dulbecco's modified Eagle's medium-low glucose; RT-PCR, reverse transcription-polymerase chain reaction; RIPA, radio-immunoprecipitation assay; SDS-PAGE, sodium dodecyl sulfate-polyacrylamide gel electrophoresis; PVDF, polyvinylidene fluoride; TBST, Tris-buffered saline Tween-20

Key words: transforming growth factor- $\beta 1$, Smad3, Smad7, human umbilical cord mesenchymal stem cells, hepatic stellate cells compared with the negative control group at 24 and $48 \mathrm{~h}$ $(\mathrm{p}<0.05)$. Apoptosis of HSCs in the co-culture group increased compared with that in the negative control group, which was more obvious at $48 \mathrm{~h}(\mathrm{p}<0.05)$. The concentration of TGF- $\beta 1$ in the co-culture group was significantly lower than in the HSCs cultured alone $(\mathrm{p}<0.05)$. After HSCs were co-cultured with hUC-MSCs for $48 \mathrm{~h}$, expression of TGF- $\beta 1$ and Smad3 mRNA and protein was reduced and expression of Smad7 mRNA and protein was increased compared with the negative control group $(\mathrm{p}<0.05)$. hUC-MSCs inhibited proliferation of HSCs, possibly through inhibiting TGF- $\beta 1$ and Smad 3 expression and increasing Smad7 protein expression.

\section{Introduction}

Viral hepatitis affects millions of people worldwide, and is now the leading cause of cirrhosis in China. Cirrhosis is a pathological manifestation of end-stage liver disease that contributes significantly to the high mortality of liver diseases. The most important part in the formation of hepatic fibrosis is extracellular matrix (ECM) deposition from hepatic stellate cells (HSCs) (1). HSCs are the final target cells in all types of liver fibrosis. HSCs are activated and transformed into muscle fibroblast-like cells, which causes increased synthesis and degradation of collagen (2).

At present, orthotropic liver transplantation is the last resort for the treatment of cirrhosis. However, due to the shortage of donor organs, orthotropic liver transplantation is restricted clinically (3), and alternative treatment strategies are urgently needed.

Stem cell therapy as a potential therapeutic method has attracted increased attention. Mesenchymal stem cells (MSCs) are important members of the stem cell family. They have the characteristics of multi-differentiation potential, hematopoietic support and promotion of stem cell transplantation, immune regulation and self replication (4). Human umbilical cord MSCs (hUC-MSCs) are derived from umbilical cord tissue. Aspiration of MSCs does not require invasive procedures, which differs from bone marrow MSCs (BM-MSCs). hUC-MSCs are routinely discarded after delivery, without ethical consideration. There have been few studies on the treatment of liver fibrosis with tissue-derived MSCs (umbilical cord, placenta, or adipose tissue), and their role in the 
treatment of liver fibrosis has rarely been compared with that of hUC-MSCs and BM-MSCs. MSCs can secrete various cytokines in a paracrine manner, which can promote liver repair, such as hepatocyte growth factor (5), while others can inhibit the occurrence of liver cirrhosis (6).

A recent investigation found that hUC-MSCs can accelerate the resolution of acute liver injury without any differentiation and manipulation (7). Additionally, it has been confirmed histologically that transplantation of hUC-MSCs into rats with $\mathrm{CCl}_{4}$-induced liver fibrosis results in significant reduction of liver fibrosis (8). However, to the best of our knowledge, there are few studies concerning the mechanism of action of hUC-MSCs on HSCs, and what type of signal transduction pathways are used in HSCs.

Transforming growth factor- $\beta 1$ (TGF- $\beta 1$ ) is a key member of the TGF- $\beta$ superfamily and plays a critical role in the development of hepatic fibrosis. The Smads protein family is located on HSCs and is divided into receptor activation and inhibitory Smads, such as Smad3 and Smad7. TGF- $31 /$ Smads is an important signaling pathway in hepatic fibrosis (9). In this study, we confirmed that hUC-MSCs could inhibit proliferation of HSCs, and clarified the effect on the TGF- $\beta 1 / \mathrm{Smads}$ pathway when hUC-MSCs were co-cultured with HSCs. This will provide a theoretical basis for the therapeutic use of MSCs in the treatment of liver fibrosis.

\section{Materials and methods}

Materials. Human HSC cell line LX2 was kindly gifted by Scott L. Friedman, Mount Sinai School of Medicine, New York, NY, USA. We used Dulbecco's modified Eagle's medium (DMEM)-low glucose (LG) culture medium and fetal bovine serum (FBS; HyClone, Logan, UT, USA), 3-(4,5-dimethylthiazol-2-yl)-2,5-diphenyltetrazolium bromide (MTT) reagent cartridge (Sigma, St. Louis, MO, USA), TGF- $\beta 1$ enzyme-linked immunosorbent assay (ELISA) reagent cartridge (R\&D Systems, Minneapolis, MN, USA), human TGF- $\beta 1$ and $\beta$-actin antibody (Bioworld, Minneapolis, MN, USA), human Smad7 antibody (Abcam, Hong Kong, China), horseradish-peroxidase-labeled goat anti-rabbit IgG (Bioworld), RNA extraction reagent RNAiao Plus, reverse transcription kit (both from Takara Biotechnology, Dalian, Japan), ECL light kit (Thermo Fisher Scientific, Inc., Waltham, USA), polyvinylidene fluoride (PVDF) membrane (Millipore, Billerica, MA, USA), semipermeable Transwell insert film (Corning Costar, Acton, MA, USA), Hoechst 33324 dye (Sigma), BCA protein concentration assay kit (Beijing Soledad Bao Biological Technology Co., Ltd., Beijing, China), and protein lysate (Biotechnology Research Institute, Haimen, China) in our experiment.

Culture of hUC-MSCs. hUC-MSC cultures were established from the umbilical cords of healthy donors using the direct plastic adherence method after informed consent had been obtained. The study was approved by the Ethics Committee of the School of Life Science and Biopharmaceutics of Lanzhou University (Lanzhou, China) and performed in accordance with the Helsinki Declaration. The cord tissue pieces were minced into 3-5-mm long fragments, plated separately in $100-\mathrm{mm}$ diameter polystyrene tissue culture dishes and main- tained in Dulbecco's modified Eagle's medium-low glucose (DMEM-LG) medium with $10 \% \mathrm{FBS}$ at $37^{\circ} \mathrm{C}$ in a humidified atmosphere with $5 \% \mathrm{CO}_{2}$. The culture medium was changed on day 7 and then every 3-4 days. Approximately 3 weeks later, when well-developed colonies of fibroblast-like cells were $80-90 \%$ confluent, the cord tissue pieces were removed and the cultures were washed and harvested with $0.25 \%$ trypsin. The cells were then seeded in new 100-mm diameter flasks for further expansion (6).

Culture of HSCs. LX2 cells were grown in DMEM-LG medium with $10 \% \mathrm{FBS}$ in $5 \% \mathrm{CO}_{2}$ at $37^{\circ} \mathrm{C}$. For all of the experiments, subconfluent cells $(80 \%)$ were incubated in $25-\mathrm{cm}^{2}$ culture bottles for different time periods ( 24 and $48 \mathrm{~h}$ ).

Establishment of co-culture system. For indirect co-culture, hUC-MSCs and LX2 cells were seeded at a 1:1 ratio in each well of a 6-well plate, using Transwell membranes ( $24 \mathrm{~mm}$ diameter, $0.4 \mu \mathrm{m}$ pore size; Corning Costar). Approximately $10^{5} \mathrm{LX} 2$ cells were placed in the lower chamber with $10^{5}$ hUC-MSCs placed on the membrane insert. Co-cultures were maintained in DMED-LG with $10 \%$ FBS for 24 or $48 \mathrm{~h}$.

The upper and lower double-cell co-culture system was established between hUC-MSCs and HSCs as the experimental group. HSCs were cultured alone as a negative control group.

Growth curve. hU-MSC and LX2 cell suspensions were inoculated in 96-well plates for 9 days. Living cells from three wells were harvested and counted serially at $24-\mathrm{h}$ intervals. Then a growth curve was plotted.

MTT assay. Cell proliferation inhibition rate of each group was evaluated by MTT assay. Cells were seeded into the aforementioned co-culture system in $2 \mathrm{ml}$ of medium in each well and cultured for 24 or $48 \mathrm{~h}$. The Transwell membranes were removed and MTT solution [5 $\mathrm{mg} / \mathrm{ml}$ in phosphate-buffered saline (PBS)] was added to each well and plates were incubated for an additional $4 \mathrm{~h}$ at $37^{\circ} \mathrm{C}$. Dimethyl sulfoxide (DMSO) was added to each well, followed by incubation on a shaker at $10 \mathrm{~min}$ at $37^{\circ} \mathrm{C}$. The liquid was transferred into 96 -well plates, $150 \mu \mathrm{l} /$ hole, 10 wells/group. Absorbance was measured on a microplate reader (Bio-Rad, Hercules, CA, USA) at $490 \mathrm{~nm}$. Cell growth inhibition rate $=(1-\mathrm{A}$ value of experimental group/control group A value) x100\% were then calculated.

Hoechst. The co-culture and control groups were cultured for 24 or 48 h. Cells were fixed with $3.7 \%$ paraformaldehyde for $30 \mathrm{~min}$ at room temperature, washed with PBS, and stained with Hoechst 33324 at a final concentration of $5 \mu \mathrm{g} / \mathrm{ml}$ at $37^{\circ} \mathrm{C}$ for 6-9 min. Cells were observed under a fluorescence microscope equipped with a UV filter. The images were recorded on a computer with a digital camera (Olympus, Takachiho, Japan) attached to the microscope, and the images were processed by computer. The Hoechst reagent was taken up by the nuclei of the cells, and apoptotic cells exhibited a bright blue fluorescence.

ELISA. TGF- $\beta 1$ protein was measured in the LX2-conditioned medium after co-culture with the hUC-MSCs for 24 or $48 \mathrm{~h}$. Serum-deprived LX2 cells served as a positive control. We used 

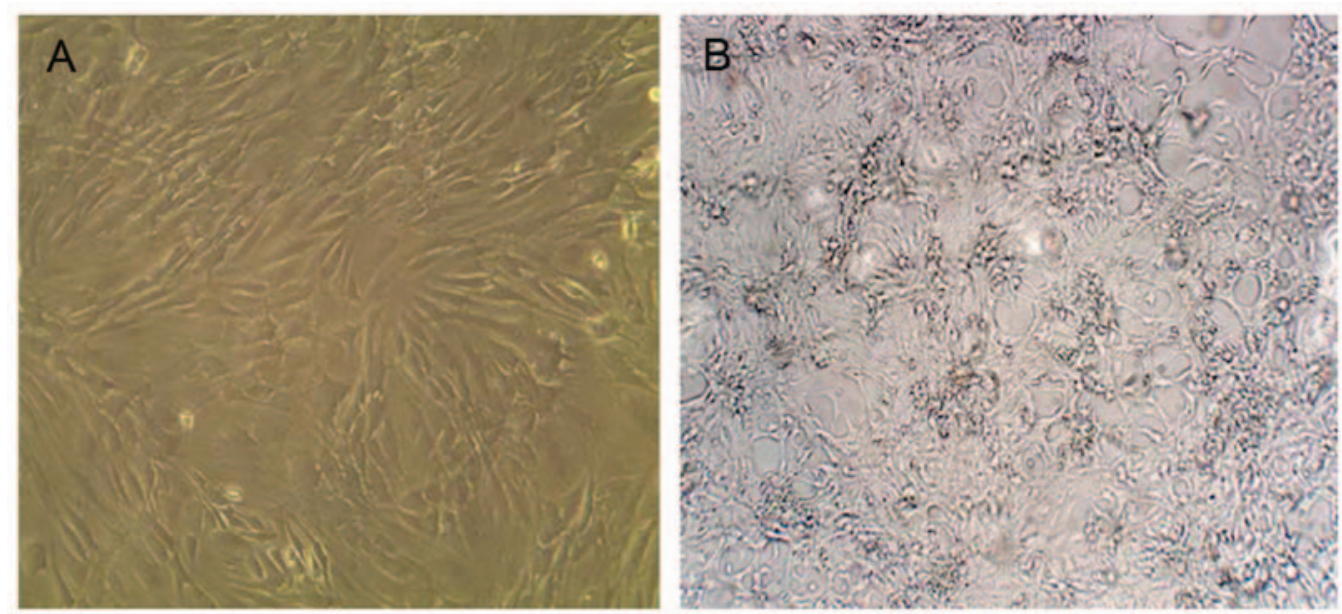

Figure 1. Morphological changes and growth of cells. (A) Human umbilical cord mesenchymal stem cells (hUC-MSCs) were cultured to $80 \%$ confluence, and became elongated and similar to fibroblasts which in parallel or spiral-like growth. (Magnification, x100). (B) LX2 cells were cultured to $80 \%$ confluence, and showed flaky stretch and pseudopodia. Filamentous structures were visible in the cells. (Magnification, x100).

a commercial ELISA kit (R\&D Systems). Reverse transcription polymerase chain reaction (RT-PCR). Total RNA was extracted from the hUC-MSCs and LX2 cells using the RNA Plus kit. TGF- $\beta 1$, primers forward, 5'-CCACAACGAAATCTATGAC-3' and reverse, 5'-GTATTTCTGGTACAGCTCCA-3; Smad3, primers forward, 5'-CTGGCTACCTGAGTGAAGATG-3' and reverse, 5'-TGTGAAGCGTGGAATGTCTC-3'; Smad7, primers forward, 5'-TCTGCGAACTAGAGTCTCCC-3' and reverse, 5'-ACGCACCAGTGTGACCGATC-3' were used for PCR. Reverse transcription was carried out using the Takara PrimeScript reagent kit with $1 \mu \mathrm{g}$ total RNA as a template and oligo(dT) as a primer. All semi-quantitative PCR experiments were performed using the same serially diluted cDNA batches as templates. Amplification was performed at $95^{\circ} \mathrm{C}$ for $30 \mathrm{sec}$ and $95^{\circ} \mathrm{C}$ for $5 \mathrm{sec}$ followed by 40 cycles of $60^{\circ} \mathrm{C}$ for $30 \mathrm{sec}$ and analyzed by fluorescence quantitative thermal cycling PCR (Bio-Rad). The PCR of human $\beta$-actin was performed as a control. TGF- $\beta 1$, Smad7 mRNA expression levels were detected. The data were calculated by the $2^{-\Delta \Delta \mathrm{CT}}$ method.

Apoptosis analysis. To detect early apoptotic changes, LX2 cells were cultured alone or co-cultured with hUC-MSCs for 24 or $48 \mathrm{~h}$, as described previously. Apoptotic cell death was detected by Annexin V/propidium iodide (PI) staining using the MEBCYTO apoptosis kit (MBL, Nagoya, Japan). LX2 cells from the various cultures were digested with $2.5 \mathrm{~g} / 1$ trypsin, harvested, washed and resuspended in $300 \mu \mathrm{l}$ binding buffer, followed by incubation with $5 \mu \mathrm{l}$ Annexin V-FITC and $5 \mu \mathrm{l}$ PI at room temperature for $15 \mathrm{~min}$ in the dark. Following incubation, $200 \mu \mathrm{l}$ binding buffer was added and the cell samples were measured using flow cytometry (BD Biosciences, New Jersey, NY, USA).

Western blotting. Cells were harvested in $0.15 \mathrm{ml}$ radioimmunoprecipitation assay (RIPA) lysis buffer with protease inhibitors and centrifuged at 12,000 rpm for $5 \mathrm{~min}$. The supernatants were assayed for protein concentration. Protein content was measured by BCA protein concentration determination kit. The sample size was $30 \mu \mathrm{g}$. Protein samples were heated at $100^{\circ} \mathrm{C}$ for $10 \mathrm{~min}$ before loading and the samples were subjected to $10 \%$ sodium dodecyl sulfate-polyacrylamide gel electrophoresis (SDS-PAGE) and transferred to PVDF membranes. Membranes were blocked with 5\% skimmed milk powder in TBST buffer $(20 \mathrm{mmol} / 1$ Tris, $500 \mathrm{mmol} / \mathrm{l}$ $\mathrm{NaCl}$, and $0.1 \%$ Tween-20) for $2 \mathrm{~h}$ at $37^{\circ} \mathrm{C}$ with gentle shaking. Membranes were incubated overnight at $4^{\circ} \mathrm{C}$ with various primary antibodies. The following primary antibodies were used: 1:1,000 rabbit polyclonal anti-TGF- $\beta 1,1: 1,000$ rabbit polyclonal anti-Smad7, and 1:1,000 rabbit polyclonal antiSmad3. The membranes were washed with TBST buffer and incubated in the appropriate peroxidase-conjugated secondary antibody solution at a 1:5,000 dilution before they were finally developed with enhanced chemiluminescence. The density of the individual bands was quantified using a densitometric scanner with Gel-pro Analyzer (Media Cybernetics, Inc., Rockville, MD, USA).

Statistical analysis. All statistical calculations were performed using GraphPad Prism software (GraphPad Software, La Jolla, $\mathrm{CA}$, USA). The data are presented as the mean $\pm \mathrm{SD}$. When applicable, Student's unpaired t-test, one-way ANOVA and Holm-Sidak test were used to determine significance. $\mathrm{p}<0.05$ was considered statistically significant.

\section{Results}

Morphological changes and growth of cells. Umbilical cords tissue block attaching to the wall was observed after 1-2 days, and some cells crawled out of the tissue block. The cultured hUC-MSCs became spindle-shaped on day 6. The cells had a long fusiform or flat shape after $\sim 10$ days. The fused cells were elongated and similar to fibroblasts that were in parallel or spirallike growth after 21 days and cell fusion was $80 \%$ (Fig. 1A). The third passage cells were for transplant spare.

LX2 cells reached $>80 \%$ cell fusion after 5-8 days. The growth of cells retained its original status without change of culture medium for 1-2 days. After 3 days, cell growth entered the logarithmic phase, and reached a plateau after 5 days (Fig. 1B). LX2 showed obvious growth and proliferation when spared 3-4 generations were used in the experiment. 


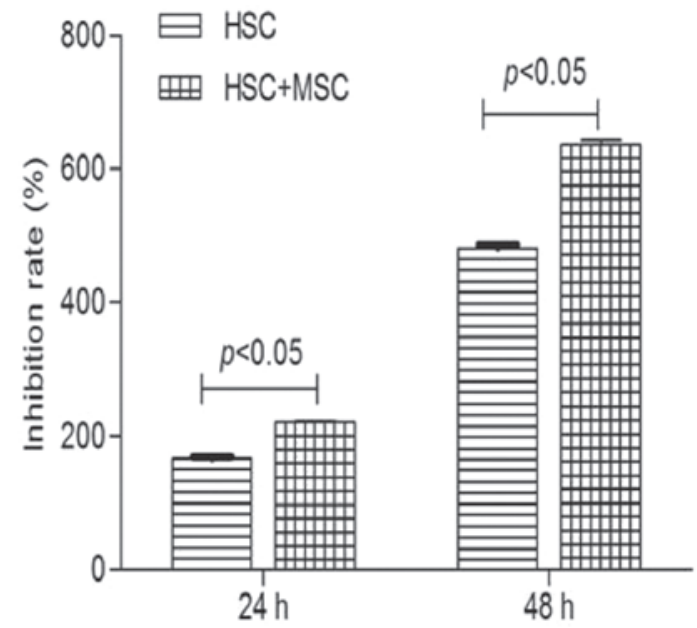

Figure 2. Inhibition of hepatic stellate cell (HSC) proliferation by human umbilical cord mesenchymal stem cells (hUC-MSCs). The inhibition rate of the experimental groups and control groups, as shown by MTT assay at 24 and $48 \mathrm{~h}$.

The upper and lower double-cell co-culture system was established between hUC-MSCs and HSCs as the experimental group. HSCs were cultured alone as a negative control group.

Inhibition of HSC proliferation by hUC-MSCs. We detected the absorbance of hUC-MSCs on HSCs by MTT assay after being cultured for 24 and $48 \mathrm{~h}$. Cell growth inhibition rates (average absorbance of each inhibited group/non-inhibited group) were then calculated. The inhibition rate of hUC-MSCs on HSCs at 24 and $48 \mathrm{~h}$ was $2.21 \pm 0.02$ and $6.37 \pm 0.06 \%$, respectively. A significant difference was observed between the co-culture experimental group $(2.21 \pm 0.02$ and $6.37 \pm 0.06 \%)$ and negative control group $(1.66 \pm 0.02$ and $4.82 \pm 0.05 \%)$ at $24 \mathrm{~h}(\mathrm{p}<0.05)$ and $48 \mathrm{~h}(\mathrm{p}<0.05)$ (Fig. 2). hUC-MSCs significantly inhibited the proliferation of HSCs in a time-dependent manner at 24 and $48 \mathrm{~h}$.

Apoptosis of HSCs induced by hUC-MSCs. After hUC-MSCs co-cultured with LX2 for 24 and $48 \mathrm{~h}$, the cells were double stained with Annexin V-FITC and PI to detect apoptosis rate of LX2 using flow cytometry. Apoptosis of LX2 cells in the co-culture system was significantly increased compared with the control group (Figs. 3 and 4). The effect of hU-MSCs on LX2 cell apoptosis was evaluated by Hoechst 33342 staining. Apoptotic cells demonstrating nuclear condensation were detected by Hoechst 33342 staining and fluorescence microscopy. As illustrated in Fig. 5, co-culture of LX2 cells and hU-MSCs for $24 \mathrm{~h}$ showed more cells with condensed and fragmented nuclei than in the negative control group. Similar results were obtained at $48 \mathrm{~h}$. The number of apoptotic bodies in the experimental group was significantly increased compared with the control group.

Decreased level of TGF- $\beta 1$ caused by hUC-MSCs. TGF- $\beta 1$ is a cytokine that plays a central role in fibrosis. To investigate whether hU-MSCs affected TGF- $\beta 1$ production in the co-culture system, the cells were subjected to ELISA to measure production of the profibrotic cytokine TGF- $\beta 1$ by LX2. TGF- $\beta 1$ protein in LX2 was significantly decreased in the co-culture group compared with the negative control group at $24 \mathrm{~h}$, with similar results at $48 \mathrm{~h}(\mathrm{p}<0.05)$ (Fig. 6).

The expression of TGF- $\beta 1$, Smad3 and Smad7 mRNA. TGF- $\beta 1$, Smad3 and Smad7 mRNA expression in LX2 cells was determined by RT-PCR. After 24 h co-culture, TGF- $\beta 1$
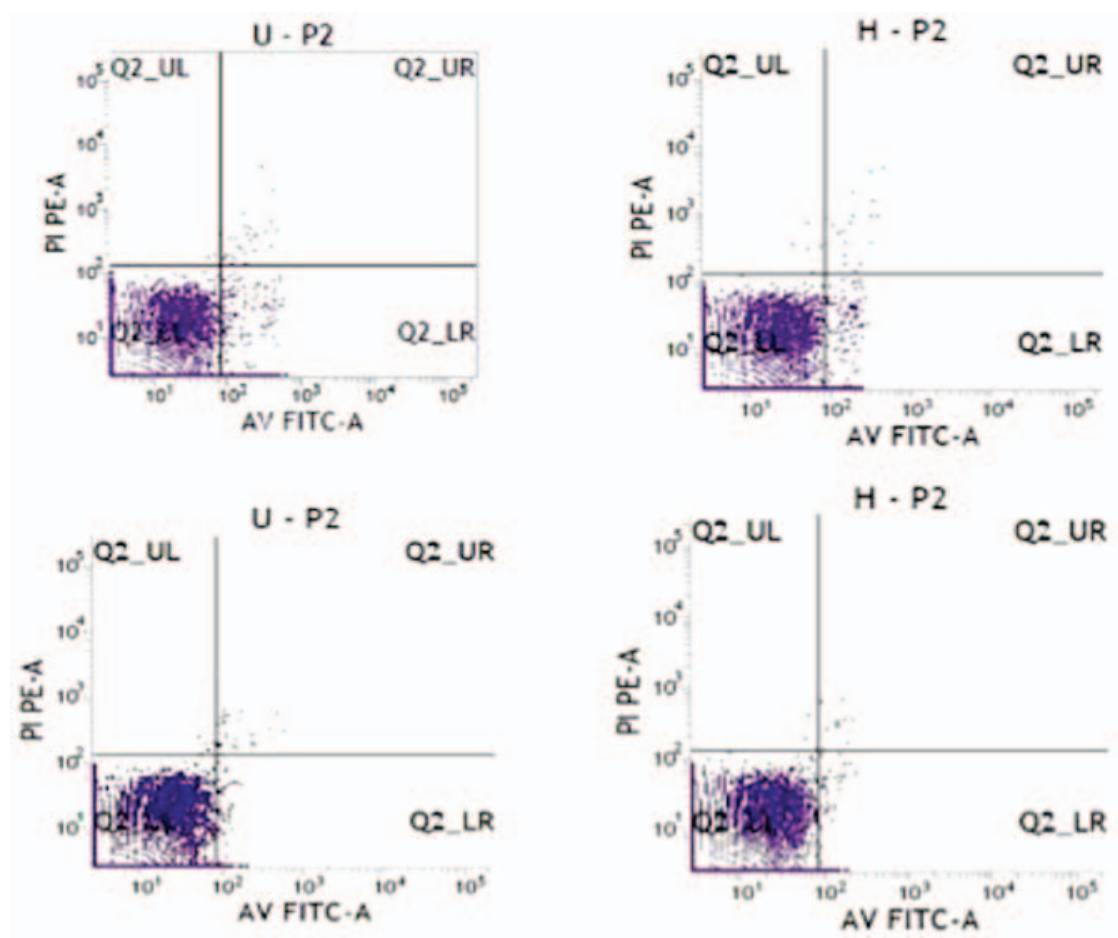

Figure 3. Apoptosis of hepatic stellate cells (HSCs) induced by human umbilical cord mesenchymal stem cells (hUC-MSCs). (A) Apoptosis rate of experimental group at $24 \mathrm{~h}$. (B) Apoptosis rate of control group at $24 \mathrm{~h}$. (C) Apoptosis rate of experimental group at $48 \mathrm{~h}$. (D) Apoptosis rate of control group at $48 \mathrm{~h}$. 
A

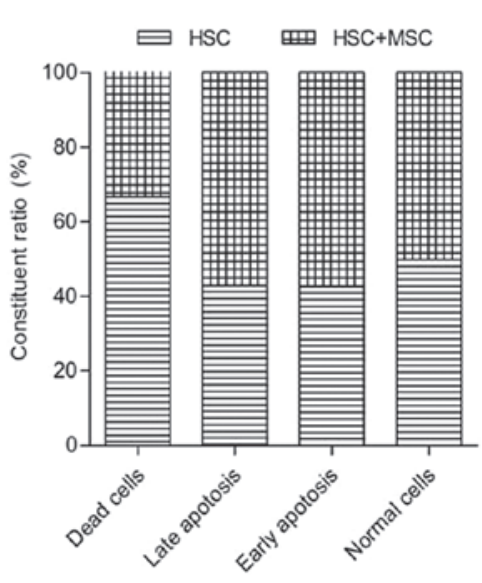

C

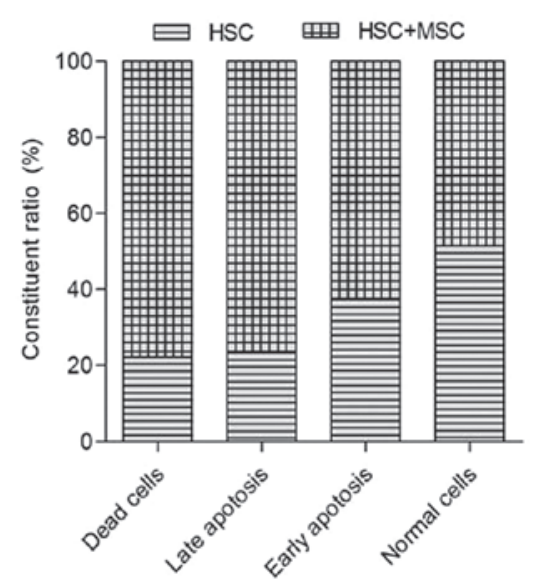

B $\square$ Normal cells

Apoptosis cells

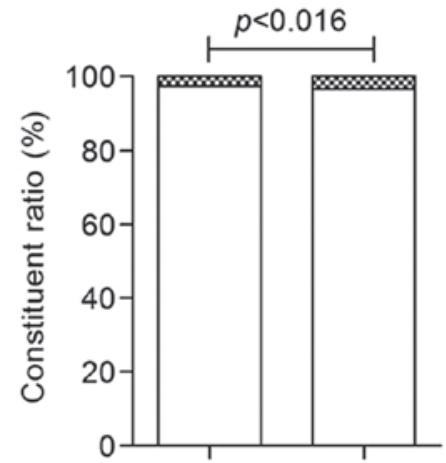

$x^{0}$

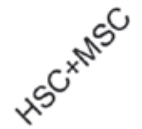

D

$\square$ Normal cells

Apoptosis cells

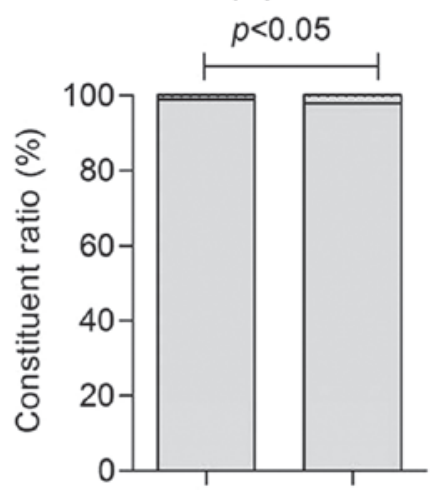

$\lambda^{c}$

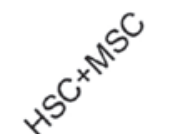

Figure 4. Apoptosis of LX2. (A) Apoptotic cell level in the co-culture system was significantly increased than compared with that in the control group at $24 \mathrm{~h}$. (B) The results had significant difference at $24 \mathrm{~h}$. (C) Apoptotic cell level in the co-culture system was significantly increased than compared with that in the control group at $48 \mathrm{~h}$. (D) The results had significant difference at $48 \mathrm{~h}$.
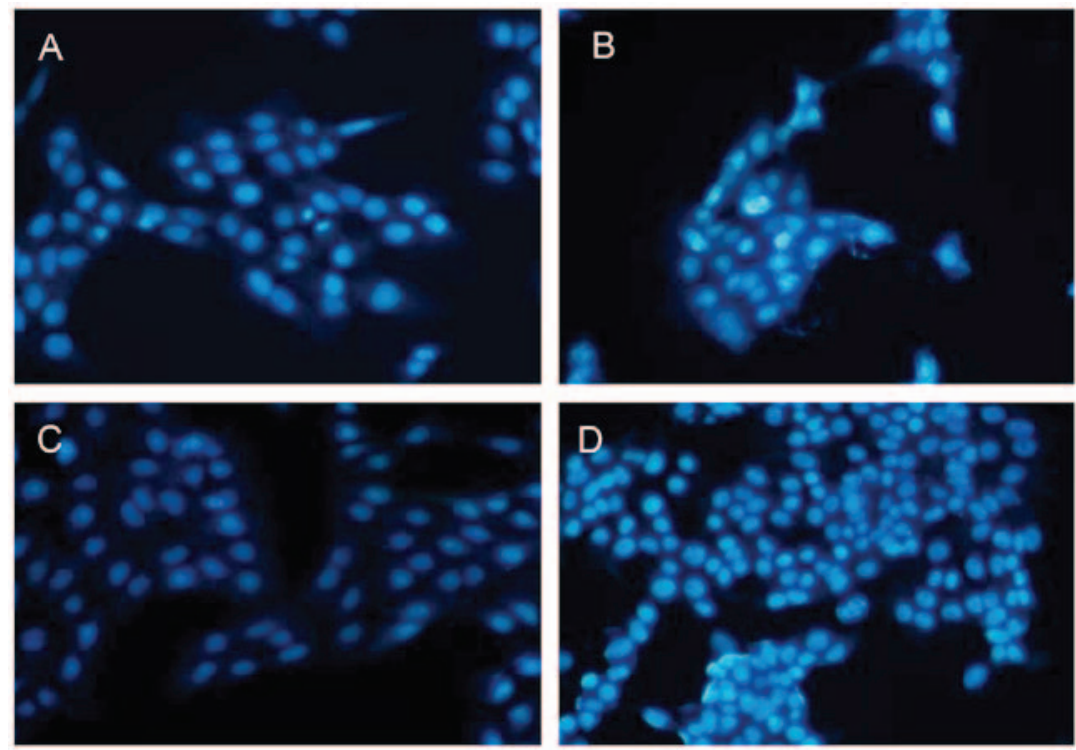

Figure 5. Hoechst results. (A) Control group at 24 h. (B) Experimental group at 24 h. (C) Control group at 48 h. (D) Experimental group at 48 h. (Magnification, $\mathrm{x} 100)$. 


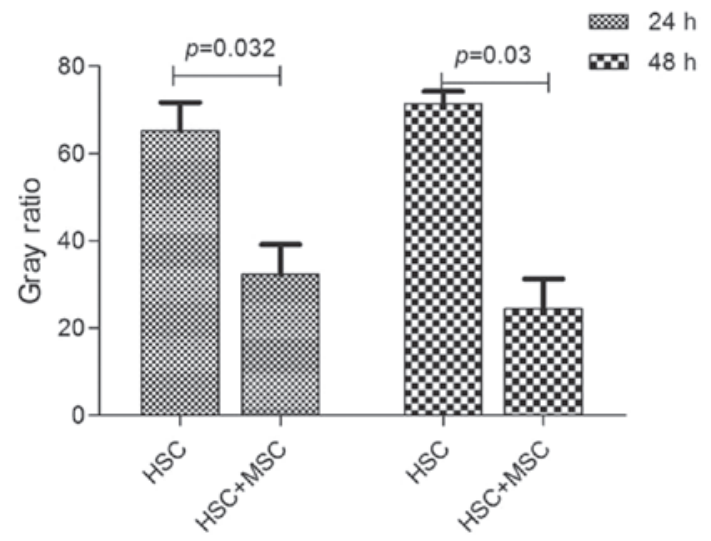

Figure 6. Decreased level of transforming growth factor- $\beta 1$ (TGF- $\beta 1$ ) caused by human umbilical cord mesenchymal stem cells( hUC-MSCs). TGF- $\beta 1$ protein in hepatic stellate cells (HSCs) was significantly decreased in co-culture groups compared with control group at $24 \mathrm{~h}$, and with similar results at $48 \mathrm{~h}$.

and Smad3 mRNA expression in the experimental group was significantly lower than that in the negative control group $(\mathrm{p}<0.05)$, but Smad7 mRNA expression increased compared

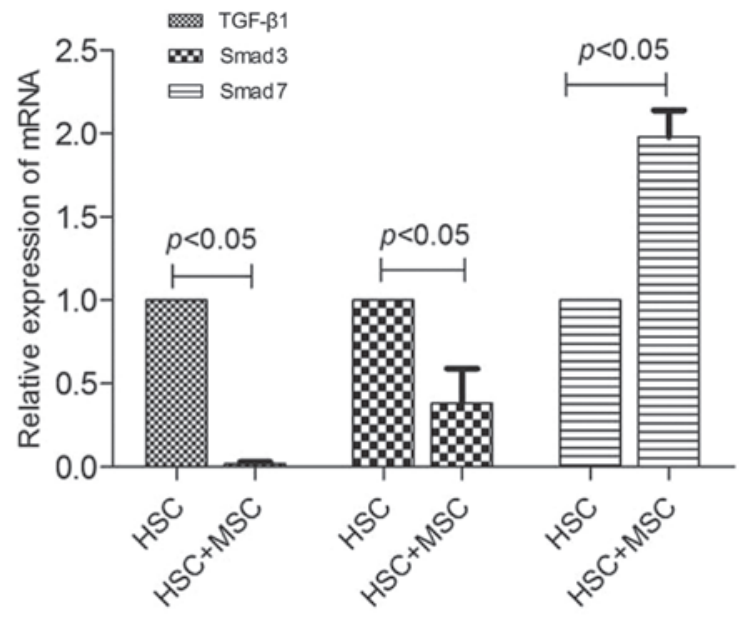

Figure 7. The expression of transforming growth factor- $\beta 1$ (TGF- $\beta 1$ ), Smad3 and Smad7 mRNA.

A

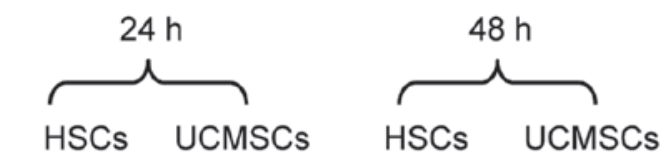

TGF- $\beta 1$

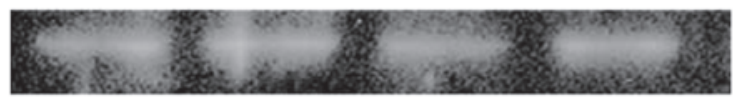

Smad 3

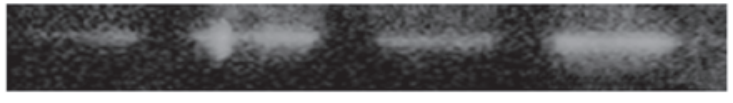

Smad 7

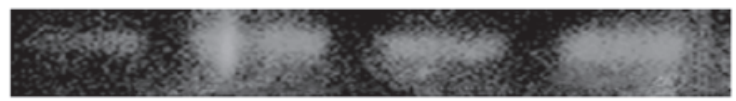

$\beta$-actin
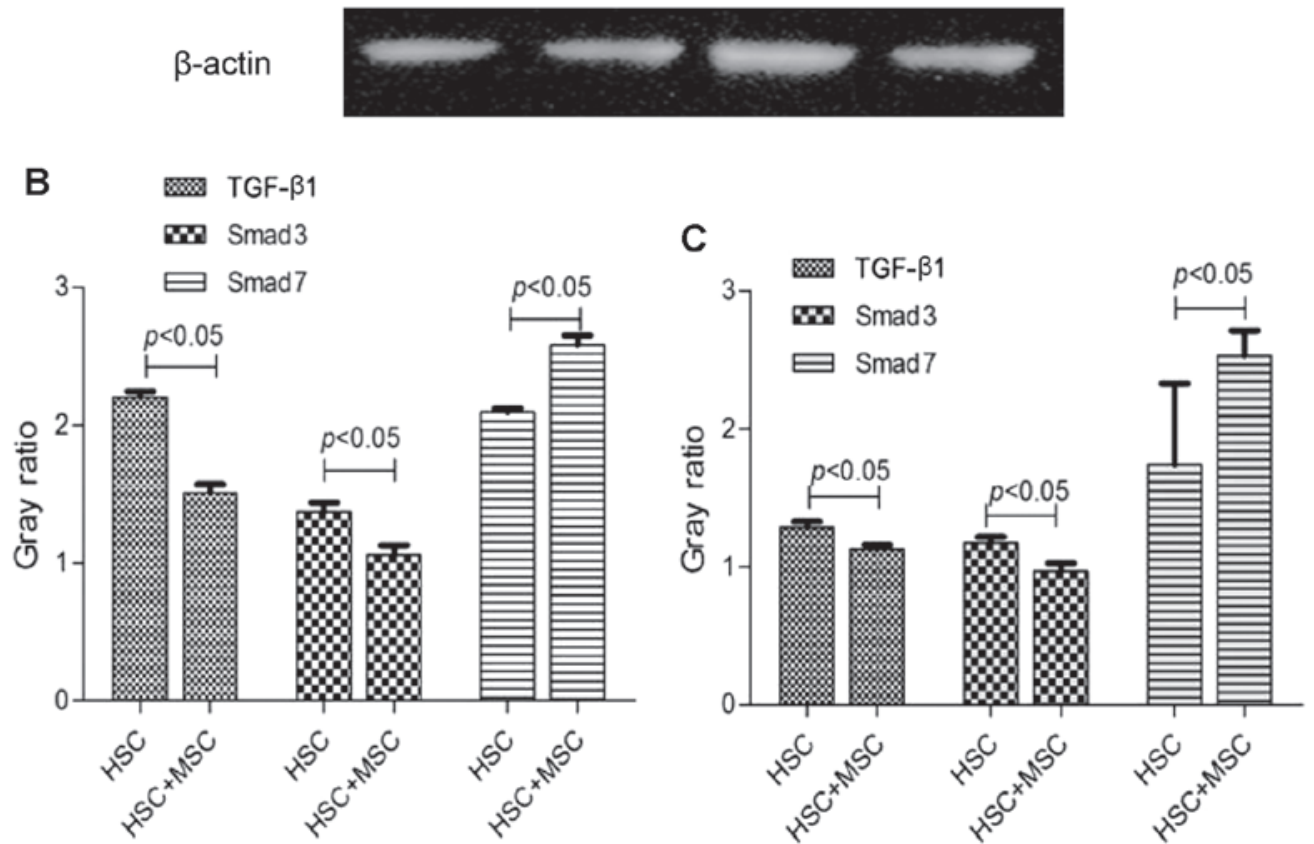

Figure 8. Expression of transforming growth factor- $\beta 1$ (TGF- $\beta 1$ ), Smad3 and Smad7 protein in control group and experimental group. (A) From left to right: control group at $24 \mathrm{~h}$, experimental group at $24 \mathrm{~h}$, control group at $48 \mathrm{~h}$, the experimental group at $48 \mathrm{~h}$. (B) The expression of TGF- $\beta 1$, Smad3 and Smad7 protein at $24 \mathrm{~h}$. (C) The expression of Smad3 and Smad7 protein at $48 \mathrm{~h}$. 
Protein expression of TGF- $\beta 1$, Smad3 and Smad7 by $h U C-M S C s$. After the hUC-MSCs were co-cultured with LX2 cells for $24 \mathrm{~h}, \mathrm{TGF}-\beta 1$ and Smad3 protein began to decrease, and their expression $(1.51 \pm 0.06$ and $1.06 \pm 0.07)$ was significantly lower than that in the control group $(2.20 \pm 0.04$ and $1.37 \pm 0.07)(\mathrm{p}<0.05)$. Similar results were found at $48 \mathrm{~h}$, their expression $(1.13 \pm 0.03$ and $0.97 \pm 0.06)$ was significantly lower than that in the control group $(1.29 \pm 0.04$ and $1.18 \pm 0.04)$ $(\mathrm{p}<0.05)$. After 24 and $48 \mathrm{~h}$ co-culture, Smad7 protein expression in the experimental group $(2.58 \pm 0.07$ and $2.35 \pm 0.18)$ increased significantly compared with that in the control group $(2.09 \pm 0.13$ and $1.74 \pm 0.59)(\mathrm{p}<0.05)($ Fig. 8).

\section{Discussion}

Our study is believed to be the first to show that hUC-MSCs inhibit the proliferation of HSCs by affecting the TGF- $\beta / \mathrm{Smads}$ pathway, and the formation of liver fibrosis. It is well known that HSCs are the main source of ECM, and the proliferation and activation of HSCs may promote the occurrence of hepatic fibrosis. It has been shown previously that the MSCs may be affected by the signal transduction pathway during activation of HSCs (10), but the effect of hUC-MSCs on HSCs is not clear. This study confirmed that using an in vitro model, hUC-MSCs could inhibit proliferation of HSCs by inhibiting the TGF- $\beta /$ Smads pathway.

Prior to this study, we used BM-MSCs to inhibit liver fibrosis (11). However, compared with BM-MSCs, hUC-MSCs are more primitive, have greater differentiation ability and low immunogenicity, are not subject to ethical constraints, and are easy to culture through a noninvasive procedure. Røsland et al (12) reported a $45.8 \%$ rate of spontaneous malignant transformation during culture of BM-MSCs. It has been suggested that spontaneous malignant transformation represents a biohazard in long-term ex vivo expansion of BM-MSCs, but hUC-MSCs propagating in continuous culture ultimately enter senescence and are not susceptible to spontaneous malignant transformation (13). Therefore, we chose hUC-MSCs for this study. We separated the hUC-MSCs from the umbilical cord for culture, and verified their ability to differentiate into fat cells in vitro.

The proliferation and activation of HSCs is an important step in the development of hepatic fibrosis (14). We would like to understand further whether hUC-MSCs can inhibit the proliferation of HSCs by regulating their proliferation. We used Transwell migration assay to co-culture hUC-MSCs and HSCs, and confirmed that hUC-MSCs inhibited the proliferation of HSCs. We then used the the flow cytometry technique to verify that the increase in HSC inhibition was the result of apoptosis of hUC-MSCs and not the result of death of HSCs. Finally, we directly observed apoptosis of HSCs in co-culture with hUC-MSCs by Hoechst staining. At the same time, we concluded that these effects were not through direct contact among cells, but rather by cytokines secreted into the culture medium. ELISA showed that hUC-MSCs secreted low levels of TGF- $\beta 1$, while HSCs secreted a large amount, and the TGF- $\beta 1$ levels of co-cultured HSCs were significantly decreased. These results are similar to those reported previously in a study of low levels of TGF- $\beta 1$ in liver cirrhosis (15).
Previous studies have indicated that hUC-MSC therapy results in significant improvement of liver function and hepatic fibrosis, but the specific mechanism is still unclear.

Liver fibrosis is related to gene expression of many cytokines, such as TGF- $\beta$, platelet-derived growth factor, endothelin, fibroblast growth factor, connective tissue growth factor and leptin, and there are multiple signaling pathways involved in the formation of liver fibrosis (16-19). TGF- $\beta$ is a cytokine that causes hepatic fibrosis and plays an important role in the activation of muscle fibroblasts. It has been shown that, in the 6 weeks after $\mathrm{CCl}_{4}$-induced liver fibrosis, TGF- $\beta 1$ levels in the serum and liver increase (9). The TGF- $\beta /$ Smads signal transduction pathway is the most important in liver fibrosis, therefore, we are also interested in the effects of hUC-MSCs on the pathway. The Smads protein family is located on HSCs and is divided into receptor activation and inhibitory Smads. The former includes Smad3, which can transfer the signal from the cytoplasm to the nucleus, and promote formation of liver fibrosis. Inhibitory Smads include Smad7, which can inhibit the formation of Smads complexes and the signal transduction process, thereby inhibiting the formation of fibrosis (20). Many studies have shown that activation of the TGF- $\beta /$ Smads signaling pathway can induce collagen deposition (21). Our experiments found that the levels of TGF- $\beta 1$ and Smad 2 secreted by HSCs decreased after co-culture, while the concentration of Smad7 increased. These data confirmed that hUC-MSCs inhibit the TGF- $\beta 1 /$ Smads pathway to inhibit proliferation and promote apoptosis of HSCs, thereby inhibiting the formation of liver fibrosis.

There are still a few issues to resolve. For example, will similar results be obtained in vivo. Previous studies have shown that inhibition of the TGF- $\beta 1 /$ Smad pathway may lead to tumor occurrence (22). This needs further research.

In conclusion, this study is believed to be the first to demonstrate hUC-MSCs inhibit proliferation and induce apoptosis of HSCs by paracrine inhibition of the TGF- $\beta 1 /$ Smads pathway. Our results indicate the potential of hUC-MSCs as a method for the treatment of liver fibrosis. However, the complexity of the mechanism requires further study.

\section{Acknowledgements}

Not applicable.

\section{Funding}

This study was supported by the Natural Science Foundation of Gansu Province, China (grant no. 1506RJZA263) and the Major Projects of Science and Technology of Gansu Province, China (grant no. 1302FKDA029).

\section{Availability of data and material}

The datasets used and/or analyzed during the current study are available from the corresponding author on reasonable request.

\section{Authors' contributions}

LTZ and JFL conceived and designed the experiments. XBP and XQF performed the experiments. XBP, HC and XRM analyzed the data. XBP wrote the paper. 


\section{Ethics approval and consent to participate}

The study protocol was conducted in accordance with the provisions of the Declaration of Helsinki, 1975 and approved by the Institutional Review Board of the First Hospital of Lanzhou University.

\section{Consent for publication}

Not applicable.

\section{Competing interests}

The authors declare that they have no competing interests.

\section{References}

1. Volarevic V, Nurkovic J, Arsenijevic N and Stojkovic M: Concise review: Therapeutic potential of mesenchymal stem cells for the treatment of acute liver failure and cirrhosis. Stem Cells 32: 2818-2823, 2014.

2. Berardis S, Dwisthi Sattwika P, Najimi M and Sokal EM: Use of mesenchymal stem cells to treat liver fibrosis: Current situation and future prospects. World J Gastroenterol 21: 742-758, 2015.

3. Zhang L, Ye JS, Decot V, Stoltz JF and de Isla N: Research on stem cells as candidates to be differentiated into hepatocytes. Biomed Mater Eng 22: 105-111, 2012.

4. Secunda R, Vennila R, Mohanashankar AM, Rajasundari M, Jeswanth S and Surendran R: Isolation, expansion and characterisation of mesenchymal stem cells from human bone marrow, adipose tissue, umbilical cord blood and matrix: A comparative study. Cytotechnology 67: 793-807, 2015.

5. Berardis S, Lombard C, Evraerts J, El Taghdouini A, Rosseels V, Sancho-Bru P, Lozano JJ, van Grunsven L, Sokal E and Najimi M: Gene expression profiling and secretome analysis differentiate adult-derived human liver stem/progenitor cells and human hepatic stellate cells. PLoS One 9: e86137, 2014.

6. Pan RL, Wang P, Xiang LX and Shao JZ: Delta-like 1 serves as a new target and contributor to liver fibrosis down-regulated by mesenchymal stem cell transplantation. J Biol Chem 286: 12340-12348, 2011.

7. Burra P, Arcidiacono D, Bizzaro D, Chioato T, Di Liddo R Banerjee A, Cappon A, Bo P, Conconi MT, Parnigotto PP, et al: Systemic administration of a novel human umbilical cord mesenchymal stem cells population accelerates the resolution of acute liver injury. BMC Gastroenterol 12: 88, 2012.

8. Sakaida I, Terai S, Yamamoto N, Aoyama K, Ishikawa T, Nishina $\mathrm{H}$ and Okita $\mathrm{K}$ : Transplantation of bone marrow cells reduces $\mathrm{CCl}_{4}$-induced liver fibrosis in mice. Hepatology 40 : 1304-1311, 2004.

9. Li T, Yan Y, Wang B, Qian H, Zhang X, Shen L, Wang M, Zhou Y, Zhu W, Li W, et al: Exosomes derived from human umbilical cord mesenchymal stem cells alleviate liver fibrosis. Stem Cells Dev 22: 845-854, 2013.
10. Eom YW, Shim KY and Baik SK: Mesenchymal stem cell therapy for liver fibrosis. Korean J Intern Med 30: 580-589, 2015.

11. Zhang LT, Fang XQ, Chen QF, Chen H, Xiao P, Peng XB, Zhang SX, Li JF and Mao XR: Bone marrow-derived mesenchymal stem cells inhibit the proliferation of hepatic stellate cells by inhibiting the transforming growth factor $\beta$ pathway. Mol Med Rep 12: 7227-7232, 2015.

12. Røsland GV, Svendsen A, Torsvik A, Sobala E, McCormack E, Immervoll H, Mysliwietz J, Tonn JC, Goldbrunner R, Lønning PE, et al: Long-term cultures of bone marrow-derived human mesenchymal stem cells frequently undergo spontaneous malignant transformation. Cancer Res 69: 5331-5339, 2009.

13. Tang Q, Chen Q, Lai X, Liu S, Chen Y, Zheng Z, Xie Q, Maldonado M, Cai Z, Qin S, et al: Malignant transformation potentials of human umbilical cord mesenchymal stem cells both spontaneously and via 3-methycholanthrene induction. PLoS One 8: e81844, 2013.

14. Wang Y, Yu X, Chen E and Li L: Liver-derived human mesenchymal stem cells: A novel therapeutic source for liver diseases. Stem Cell Res Ther 7: 71, 2016.

15. Kim WH, Matsumoto K, Bessho K and Nakamura T: Growth inhibition and apoptosis in liver myofibroblasts promoted by hepatocyte growth factor leads to resolution from liver cirrhosis. Am J Pathol 166: 1017-1028, 2005.

16. Zhao YL, Zhu RT and Sun YL: Epithelial-mesenchymal transition in liver fibrosis. Biomed Rep 4: 269-274, 2016.

17. Borkham-Kamphorst E, Herrmann J, Stoll D, Treptau J, Gressner AM and Weiskirchen R: Dominant-negative soluble PDGF-beta receptor inhibits hepatic stellate cell activation and attenuates liver fibrosis. Lab Invest 84: 766-777, 2004.

18. Saxena NK, Titus MA, Ding X, Floyd J, Srinivasan S, Sitaraman SV and Anania FA: Leptin as a novel profibrogenic cytokine in hepatic stellate cells: Mitogenesis and inhibition of apoptosis mediated by extracellular regulated kinase (Erk) and Akt phosphorylation. FASEB J 18: 1612-1614, 2004.

19. Lang T, Ikejima K, Yoshikawa M, Enomoto N, Iijima K, Kitamura T, Takei Y and Sato N: Leptin facilitates proliferation of hepatic stellate cells through up-regulation of platelet-derived growth factor receptor. Biochem Biophys Res Commun 323: 1091-1095, 2004.

20. Hata A and Chen YG: TGF- $\beta$ signaling from receptors to Smads. Cold Spring Harb Perspect Biol 8: a022061, 2016.

21. Argentou N, Germanidis G, Hytiroglou P, Apostolou E, Vassiliadis T, Patsiaoura K, Sideras P, Germenis AE and Speletas M: TGF- $\beta$ signaling is activated in patients with chronic HBV infection and repressed by SMAD7 overexpression after successful antiviral treatment. Inflamm Res 65: 355-365, 2016.

22. Lu B, Zhou YN, Li Q, Wu ZQ, Zhang ZY, Ji R, Guo QH and Liu W: Correlations of TGF-betaRII, Smad4 and Smad7 expression to clinicopathologic characteristics and prognosis of gastric cancer. Ai Zheng 28: 538-542, 2009 (In Chinese).

This work is licensed under a Creative Commons Attribution-NonCommercial-NoDerivatives 4.0 International (CC BY-NC-ND 4.0) License. 